\title{
Photoreflectance Study on the Interface of InGaP/GaAs Heterostructures Grown by Gas Source Molecular Beam Epitaxy
}

\author{
Chih-Ming LAI, Fu-Yu CHANG ${ }^{1}$, Hao-Hsiung LIN ${ }^{1}$ and Gwo-Jen JAN ${ }^{1}$ \\ Department of Electronic Engineering, Ming Chuan University, Taoyuan 333, Republic of China \\ ${ }^{1}$ Department of Electrical Engineering, National Taiwan University, Taipei 107, Republic of China
}

(Received April 17, 2003; accepted October 28, 2003; published February 10, 2004)

\begin{abstract}
The photoreflectance spectra of an undoped InGaP film grown on a GaAs (001) substrate by gas-source molecular beam epitaxy have been measured at the temperature of $70 \mathrm{~K}$. The band-gap optical transitions attributed to the InGaP epilayer and GaAs substrate have been characterized. Two broad features, which originate from the interface of the InGaP and GaAs layer, also appeared with the transition energies of $1.523 \mathrm{eV}$ and $1.877 \mathrm{eV}$ in the photoreflectance spectra. In addition, the amplitudes of these two optical features show different trends with increasing power of an extra DC pumping laser. The results suggest that the two unusual optical features are attributed to the spatial indirect transitions at the interface.
\end{abstract}

[DOI: 10.1143/JJAP.43.735]

KEYWORDS: photoreflectance, InGaP/GaAs, gas-source MBE, polarization, composition modulation

\section{Introduction}

The InGaP lattice matched to GaAs with larger band-gap energies has attracted much attention because it offers the possibility of growing strain layer superlattice structures for applications of visible light emitting devices and high-speed electronic devices. ${ }^{1)}$ It also was demonstrated that InGaP has ultralow interface recombination velocities with GaAs. ${ }^{2}$ The spontaneous long-range ordering and lateral compositional modulation of InGaP material have been proven to significantly affect the electronic properties of the $\mathrm{InGaP} / \mathrm{GaAs}$ interface. Theoretically, Froyen et al. presented that the band alignment of the InGaP and GaAs heterojunction is changed from type I to type II as the InGaP becomes more ordered. ${ }^{3)}$ From the experimental results, it is seen that the conduction band offset $\left(\Delta E_{\mathrm{c}}\right)$ may spread from 30 to $220 \mathrm{meV}$ using various growth methods and conditions. ${ }^{4,5)}$ The latter effect is seen to lead to a band-gap reduction and dominate over $\mathrm{Cu}-\mathrm{Pt}$ long range ordering on determining the optical anisotropy for InGaP material. ${ }^{6,7)}$ Localized potential minimum will occur in the InGaP material because of its rich In composition. Therefore, these two effects could significantly modify the optical and electronic properties of the $\mathrm{InGaP} /$ GaAs interface.

Modulated spectroscopy has been shown to be an excellent technique for characterizing the optical properties of interface and layer crystals. ${ }^{8)}$ Kassel et al. had studied $\mathrm{ZnSe} / \mathrm{GaAs}$ and $\mathrm{ZnSe} / \mathrm{AlAs}$ interfaces by electrolyte electroreflectance and successfully characterized the cross-over transition at the interfaces. ${ }^{9)}$ In this letter, we present a study on the interfacial characteristics of the $\mathrm{InGaP} / \mathrm{GaAs}$ heterojunction grown by gas source molecular beam epitaxy (GSMBE) using the photoreflectance (PR) technique. Several features in the spectra of the InGaP/GaAs samples were observed, such as the band edge transitions and two broad optical transitions. From our experiments, we characterized the optical features in PR spectra and suggested that two broad transitions were spatial-indirect transitions from the interface between InGaP and GaAs layers.

\section{Experimental}

By using a VG $80 \mathrm{H}$ GSMBE system, a $1-\mu \mathrm{m}$-thick InGaP lattice-matched epilayer was grown on a GaAs(100) semi- insulated substrate with a $0.5-\mu \mathrm{m}$-thick undoped GaAs buffer layer at a growth temperature of $480^{\circ} \mathrm{C}$ and a growth rate of $1 \mu \mathrm{m} / \mathrm{h}$. We call this sample $\mathrm{S}_{1}(\mathrm{InGaP} / \mathrm{GaAs})$ for convenience in later discussions. In order to remove the heterojuction of InGaP/GaAs for comparative purposes, we glued the InGaP onto a piece of a flat glass plate and etched the GaAs with a chemical solution of $\mathrm{H}_{2} \mathrm{SO}_{4}: \mathrm{H}_{2} \mathrm{O}_{2}$ : $\mathrm{H}_{2} \mathrm{O}=10: 1: 1$. We call this sample $\mathrm{S}_{2}$ (InGaP alone). We also used a selective wet etching technique for sample $S_{1}$ to obtain a GaAs substrate and call it sample $\mathrm{S}_{3}$.

The samples were cooled to $70 \mathrm{~K}$ by a closed cycle cryostat. The pumping light source was a $543.2 \mathrm{~nm} \mathrm{He}-\mathrm{Ne}$ laser with a power density of $1 \mathrm{~mW} / \mathrm{cm}^{2}$. A mechanical chopper at a frequency of $265 \mathrm{~Hz}$ modulated the pumping light, which generated a modulated electric field on the sample surface. The probe light was emitted by a $120 \mathrm{~W}$ tungsten lamp and was dispersed by a $0.3 \mathrm{~m}$ monochromator. The optical signal of the reflected light from the probe light passed through a long-pass filter with a cut-off wavelength of $580 \mathrm{~nm}$ and was detected by a Si-PIN detector.

\section{Results and Discussion}

PR spectra at a temperature of $70 \mathrm{~K}$ for sample $S_{1}$ were measured with a polarization of the probe light parallel to [110] and [110] directions as shown in Fig. 1. In order to obtain the transition energy values and broadening parameters, the spectra were fitted by the third derivative of the

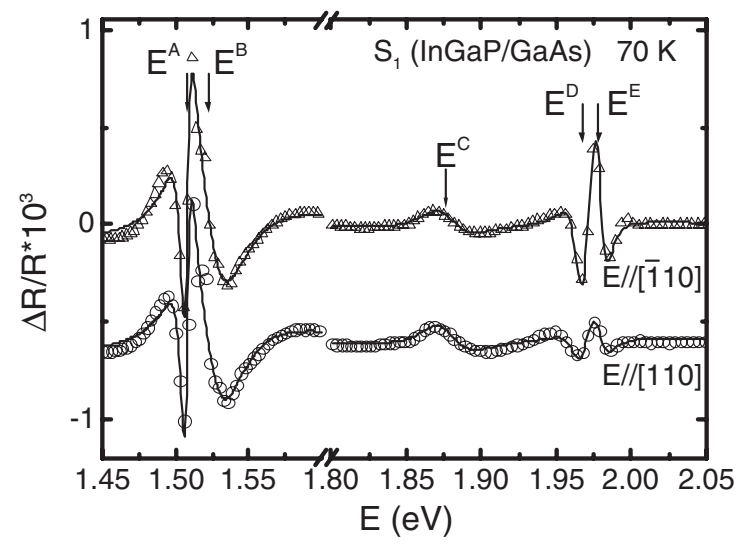

Fig. 1. Polarization-dependent PR spectra of sample $S_{1}$ at $70 \mathrm{~K}$. 
Table I. Fitting results for the PR spectra of samples $S_{1}, S_{2}$ and GaAs substrate.

\begin{tabular}{ccccc}
\hline & Sym. & Energy $(\mathrm{eV})$ & Amplitude $\left(\times 10^{4}\right)^{*}$ & $\Gamma(\mathrm{meV})$ \\
\hline $\mathrm{S}_{1}$ & $\mathrm{E}^{\mathrm{A}}$ & 1.507 & 9.1 & 6 \\
& $\mathrm{E}^{\mathrm{B}}$ & 1.523 & 4.6 & 28 \\
& $\mathrm{E}^{\mathrm{C}}$ & 1.877 & 8.6 & 26 \\
& $\mathrm{E}^{\mathrm{D}}$ & 1.967 & $3.5(1.4)$ & $10(13)$ \\
& $\mathrm{E}^{\mathrm{E}}$ & 1.978 & $4.8(1.4)$ & $8(12)$ \\
$\mathrm{S}_{2}$ & $\mathrm{E}^{\mathrm{D}}$ & 1.967 & $0.55(0.21)$ & $12(12)$ \\
& $\mathrm{E}^{\mathrm{E}}$ & 1.980 & $0.68(0.19)$ & $9(9)$ \\
$\mathrm{S}_{3}$ & $\mathrm{Eg}$ & 1.511 & 10.8 & 5 \\
& $\mathrm{Eg}+\Delta$ & 1.852 & 0.17 & 12 \\
\hline
\end{tabular}

* parenthetic values are the results for [110] polarization.

Lorentzian function lineshape. ${ }^{10)}$ In the spectra of sample $S_{1}$, five optical features ( $E^{i}, i=\mathrm{A}$ to $\mathrm{E}$ ) are observed and five arrows mark the fitted energies that are summarized in Table I. Here, we identify that the feature at $E^{\mathrm{A}}(1.507 \mathrm{eV})$ is attributed to the fundamental band-gap transitions of the GaAs substrate, while the features at $\mathrm{E}^{\mathrm{D}}(1.967 \mathrm{eV})$ and $\mathrm{E}^{\mathrm{E}}$ $(1.978 \mathrm{eV})$ are attributed to the InGaP film. ${ }^{11)}$ It is worth noting that the $\mathrm{E}^{\mathrm{D}}$ and $\mathrm{E}^{\mathrm{E}}$ features show strong anisotropy, and the amplitude ratios between polarization in the [110] and the [110] directions are 2.5 and 3.4 for the $E^{D}$ and $E^{E}$ features, respectively. According to the polarized-photoluminescence results of sample $S_{1}$ (not shown here), the PL spectrum in the [110] direction has a stronger intensity than that in the direction. ${ }^{6)}$ Thus, the origin of the anisotropic properties may be due to the lateral compositional modulation. Detailed results will be reported elsewhere. Meanwhile, two broad optical features at $\mathrm{E}^{\mathrm{B}}(1.523 \mathrm{eV})$ and $\mathrm{E}^{\mathrm{C}}$ $(1.877 \mathrm{eV})$, with broadening parameters $28 \mathrm{meV}$ and $26 \mathrm{meV}$, could be found in the spectra. The amplitudes and line shapes of the $\mathrm{E}^{\mathrm{B}}$ and $\mathrm{E}^{\mathrm{C}}$ features are almost the same for the two polarization directions.

In order to explore the origin of the $\mathrm{E}^{\mathrm{B}}$ and $\mathrm{E}^{\mathrm{C}}$ features in the spectra, we prepared the InGaP (sample $S_{2}$ ) and GaAs substrates (sample $\mathrm{S}_{3}$ ) by selective etching of the GaAs or InGaP materials from sample $S_{1}$. Displayed in Fig. 2 are the $\mathrm{PR}$ spectra of the two samples. For sample $\mathrm{S}_{2}$, only the $\mathrm{E}^{\mathrm{D}}$ and $\mathrm{E}^{\mathrm{E}}$ features appear to reflect $\mathrm{InGaP}$ band-gap transitions. Similar to sample $S_{1}$, they also show strong anisotropy. The amplitude ratios of the optical transitions in the [110] and the [110] directions are 2.6 and 3.6 for $\mathrm{E}^{\mathrm{D}}$ and $\mathrm{E}^{\mathrm{E}}$, respectively. In the PR spectrum of sample $S_{3}$ (shown in the lower part of Fig. 2), the fundamental band-gap transition and the spin orbital transition of GaAs were observed and the transition energy values are $1.511 \mathrm{eV}$ and $1.852 \mathrm{eV}$ with the broadening parameters of $5 \mathrm{meV}$ and $12 \mathrm{meV}$, respectively. ${ }^{12)}$ Compared with the $\mathrm{E}^{\mathrm{B}}$ and $\mathrm{E}^{\mathrm{C}}$ features in the PR spectra of sample $S_{1}$, we can find that the features of sample $S_{3}$ have smaller transition energies with narrower line widths and show totally different line shapes. This clearly demonstrates that the $\mathrm{E}^{\mathrm{B}}$ and $\mathrm{E}^{\mathrm{C}}$ features are not contributed by the GaAs layer. From our experimental results shown in Fig. 1 for sample $S_{1}$ (InGaP/GaAs) and Fig. 2 for samples $S_{2}$ (InGaP alone) and $\mathrm{S}_{3}$ (GaAs substrate), it is revealed that $\mathrm{E}^{\mathrm{B}}$ and $\mathrm{E}^{\mathrm{C}}$ features occur only for sample $\mathrm{S}_{1}$. Therefore, the $\mathrm{E}^{\mathrm{B}}$ and $\mathrm{E}^{\mathrm{C}}$ features must be optical transitions originating from the InGaP/GaAs interface.

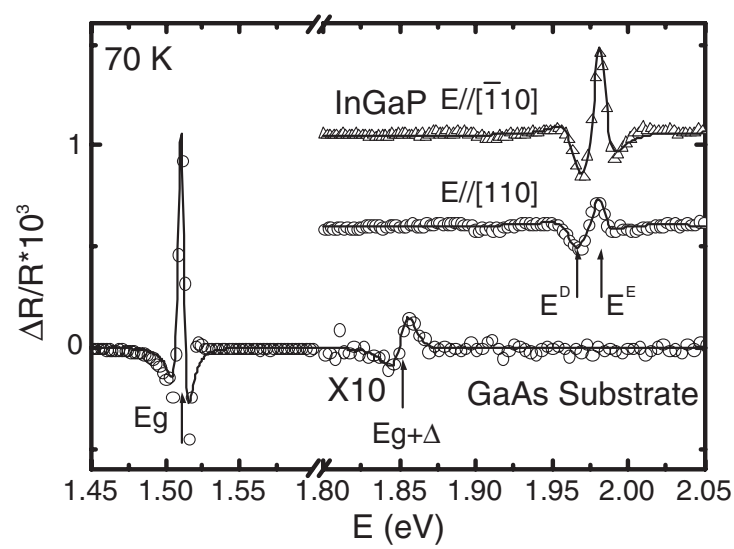

Fig. 2. PR spectra of samples $S_{2}(\mathrm{InGaP})$ and $\mathrm{S}_{3}(\mathrm{GaAs}$ substrate) at $70 \mathrm{~K}$.

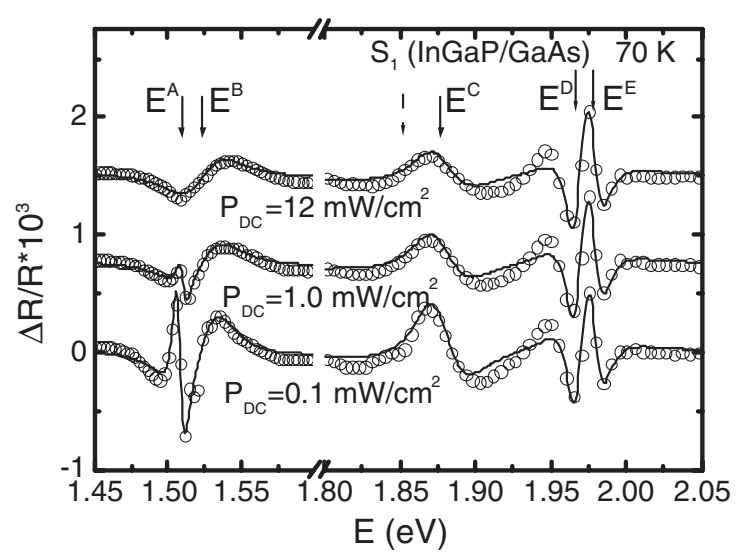

Fig. 3. PR spectra of sample $S_{1}$ at $70 \mathrm{~K}$ with extra DC pumping laser. Unit of $P_{\mathrm{DC}}$ is $\mathrm{mW} / \mathrm{cm}^{2}$.

To confirm the origin of $\mathrm{E}^{\mathrm{B}}$ and $\mathrm{E}^{\mathrm{C}}$ features in the spectra, we carefully investigate the responses of sample $\mathrm{S}_{1}$ (InGaP/ GaAs) in our PR experiments by adding an extra $786.2 \mathrm{~nm}$ DC pumping laser. As the DC pumping source is activated, the DC pumping light travels through the InGaP epilayer but is absorbed by the GaAs substrate, which generates electronhole pairs, because the photon energy of the DC pumping light is lower than the InGaP band-gap energy but higher than the GaAs band-gap energy. The photogenerated carriers can reduce the built-in electric field and downgrade the strength of the modulated electric field in the GaAs substrate due to the screening effect.

Figure 3 displays the PR spectra of sample $S_{1}$ when the $786.2 \mathrm{~nm}$ DC pumping laser is turned on. The DC pumping power densities $P_{\mathrm{DC}}$ are 12,1 and $0.1 \mathrm{~mW} / \mathrm{cm}^{2}$, respectively. Clearly, we observe that amplitudes of the optical signal of the $\mathrm{E}^{\mathrm{A}}$ feature decrease rapidly as pumping power density increases due to the screening effect in GaAs layers, while the amplitudes of the optical signal of the $\mathrm{E}^{\mathrm{D}}$ and $\mathrm{E}^{\mathrm{E}}$ features (InGaP) remain constant. Figure 4 is a plot of amplitude versus DC pumping power density $\left(P_{\mathrm{DC}}\right)$. The amplitudes of the optical signal of the $\mathrm{E}^{\mathrm{B}}$ and $\mathrm{E}^{\mathrm{C}}$ features show the same trend. Here, we calculate the ratios of $\Delta R / R$ at $P_{\mathrm{DC}}=$ $0.1 \mathrm{~mW} / \mathrm{cm}^{2}$ to $\Delta R / R$ at $P_{\mathrm{DC}}=12 \mathrm{~mW} / \mathrm{cm}^{2}$ to be $0.0,0.49$, $0.50,1.00$ and 1.02 for $\mathrm{E}^{i}(i=\mathrm{A}$ to $\mathrm{E})$ transitions, respectively. 


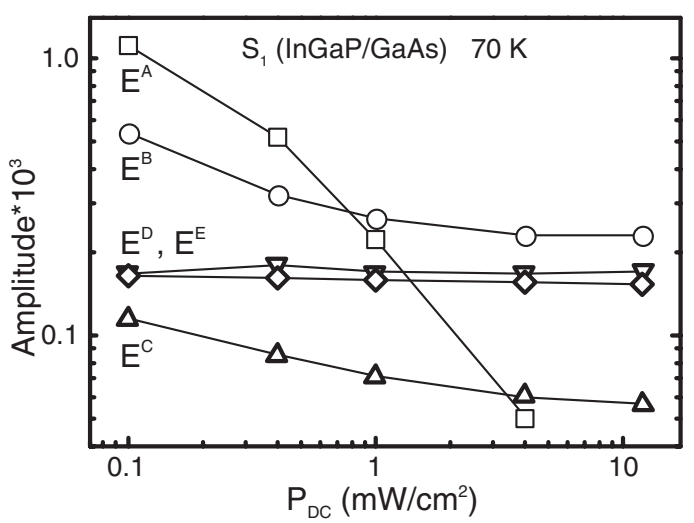

Fig. 4. Amplitudes of optical features in the PR spectra vs $P_{\mathrm{DC}}$.

The $j$-th optical feature of the PR spectrum for an $N$-layer structure could be expressed as

$$
\begin{aligned}
& \left(\frac{\Delta R}{R}\right)_{j}=\sum_{N}\left(\frac{\partial \ln R}{\partial \varepsilon_{j}^{(N)}}\right) \Delta \varepsilon_{j}^{(N)} \\
& \Delta \varepsilon_{j}^{(N)}=C_{j}^{(N)} \cdot L_{j}(E) \cdot \Delta F^{(N)} .
\end{aligned}
$$

where $L_{j}(E)$ is the line-shape function of the $j$-th optical feature and $\Delta F^{(N)}$ is the modulated electric field in Layer $N$. For the InGaP/GaAs thin film sample, eq. (1) can be written as

$$
\begin{aligned}
\left(\frac{\Delta R}{R}\right)_{j} & =\left(\frac{\partial \ln R}{\partial \varepsilon_{j}^{\mathrm{InGaP}}}\right) \Delta \varepsilon_{j}^{\mathrm{InGaP}}+\left(\frac{\partial \ln R}{\partial \varepsilon_{j}^{\mathrm{GaAs}}}\right) \Delta \varepsilon_{j}^{\mathrm{GaAs}} \\
& =\left(A_{j}^{\mathrm{InGaP}} \Delta F^{\mathrm{InGaP}}+A_{j}^{\mathrm{GaAs}} \Delta F^{\mathrm{GaAs}}\right) \cdot L_{j}(E) .
\end{aligned}
$$

It is noted that if the $j$-th optical transition does not exist in the InGaP (GaAs) layer, then $A_{j}^{\mathrm{InGaP}}\left(A_{j}^{\mathrm{GaAs}}\right)$ would be zero.

As the sample is exposed by an extra DC $786.2 \mathrm{~nm}$ laser, the modulated electric field $\left(\Delta F^{\mathrm{GaAs}}\right)$ in the GaAs substrate vanishes due to screening effect. Thus,

$$
\left(\frac{\Delta R}{R}\right)_{j}^{\mathrm{DC}}=A_{j}^{\mathrm{InGaP}} \Delta F^{\mathrm{InGaP}} \cdot L_{j}(E) .
$$

For the $j$-th optical feature, the ratio $(r)$ of amplitude with DC pumping to amplitude without DC pumping is calculated by

$$
r=\left|\frac{A_{j}^{\mathrm{InGaP}} \Delta F^{\mathrm{InGaP}}}{A_{j}^{\mathrm{InGaP}} \Delta F^{\mathrm{InGaP}}+A_{j}^{\mathrm{GaAs}} \Delta F^{\mathrm{GaAs}}}\right| .
$$

When the $j$-th optical feature is a transition in the GaAs $\left(\mathrm{E}^{\mathrm{A}}\right)$ or $\mathrm{InGaP}\left(\mathrm{E}^{\mathrm{D}}\right.$ and $\left.\mathrm{E}^{\mathrm{E}}\right)$ layer, we have $r=0$ with $A_{j}^{\mathrm{InGaP}}=0$ or $r=1$ with $A_{j}^{\mathrm{GaAs}}=0$, respectively. These values are highly consistent with our experimental results. However, for $\mathrm{E}^{\mathrm{B}}$ and $\mathrm{E}^{\mathrm{C}}$ transitions, the ratios are approximately 0.5 . Thus, both $A_{j}^{\mathrm{InGaP}} \Delta F^{\mathrm{InGaP}}$ and $A_{j}^{\mathrm{GaAs}} \Delta F^{\mathrm{GaAs}}$ should be nonzero. Therefore, the modulated laser should induce dielectric change at both sides of the interface for the $E^{B}$ and $\mathrm{E}^{\mathrm{C}}$ transitions. This means that they could absorb the photons not only on the GaAs side, but also on the InGaP side.

The band edge profiles of the $\mathrm{InGaP} / \mathrm{GaAs}$ heterojunction are shown in Fig. 5. The transitions corresponding to $\mathrm{E}^{i}(i=$ A to E) optical features are marked by arrows. The bandedge

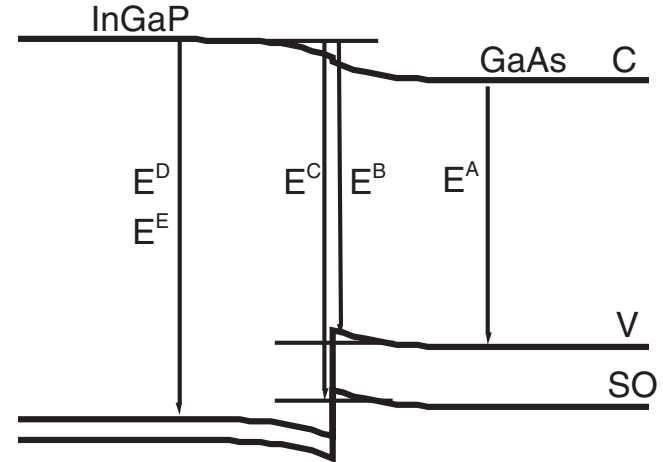

Fig. 5. Profiles of band edge for conduction band, valence band and splitoff band.

of the GaAs valence band is higher than that of InGaP. The holes must be localized in the GaAs. However, as mentioned above, the $\mathrm{E}^{\mathrm{B}}$ and $\mathrm{E}^{\mathrm{C}}$ features should have nonzero transition probabilities at both sides of the interface. Therefore, the results of extra DC pumping PR experiments suggest that the $E^{B}$ and $E^{C}$ features are due to the spatial indirect transition from the GaAs valence band to the InGaP conduction band.

\section{Conclusions}

We have studied the optical characteristics of InGaP/ GaAs grown by GSMBE at $480^{\circ} \mathrm{C}$ with the PR technique at $70 \mathrm{~K}$. The fundamental band-gap transitions of GaAs and InGaP are identified $1.507 \mathrm{eV}, 1.967 \mathrm{eV}$ and $1.978 \mathrm{eV}$. Two broader optical features $\left(\mathrm{E}^{\mathrm{B}}\right.$ and $\left.\mathrm{E}^{\mathrm{C}}\right)$ were observed in $\mathrm{PR}$ spectra located at $1.523 \mathrm{eV}$ and $1.877 \mathrm{eV}$. We used three samples, $\mathrm{S}_{1}$ (InGaP/GaAs), $\mathrm{S}_{2}$ (InGaP), and $\mathrm{S}_{3}$ (GaAs) to demonstrate that the $\mathrm{E}^{\mathrm{B}}$ and $\mathrm{E}^{\mathrm{C}}$ features originated from the InGaP/GaAs interface. Furthermore, by adding an extra DC pumping light to our PR experiments, we examined the amplitude of the signals as a function of the DC pumping power with $S_{1}$ and suggested that the $E^{B}$ and $E^{C}$ optical features are spatial indirect transitions at the interface between the InGaP layer and GaAs layer.

\section{Acknowledge}

This work was supported by the National Science Council of the Republic of China under contract No. NSC-90-2115E-002-020. We would like to thank Professor Y. H. Chen and Professor Johnson Lee for very valuable discussions.

1) G. I. Hatakoshi: Visible semiconductor lasers, ed. G. P. Agrawal (American Institute of Physics, New York, 1995) Chap. 6, p. 181.

2) J. M. Olson, R. K. Ahrenkiel, D. J. Dunlavy, Brian Keyes and A. E. Kibbler: Appl. Phys. Lett. 55 (1989) 1208.

3) S. Froyen, A. Zunger and A. Mascarenhas: Appl. Phys. Lett. 68 (1996) 2852.

4) J. Chen, J. R. Sites, I. L. Spain, M. J. Hafich and G. Y. Robinson: Appl. Phys. Lett. 58 (1991) 744.

5) M. A. Rao, E. J. Caine, H. Kroemer, S. I. Long and D. I. Babic: J. Appl. Phys. 61 (1987) 643.

6) H.-W. Ren, M. Sugisaki, S. Sugou, K. Nishi, A. Gomyo and Y. Masumoto: Jpn. J. Appl. Phys. 38 (1999) 2438.

7) M. Sugisaki, H.-W. Ren, K. Nishi, S. Sugou, and Y. Masumoto: Phys. Rev. B 61 (2000) 16040.

8) Y. C. Wang, S. L. Tyan and Y. D. Juang: J. Appl. Phys. 92 (2002) 920. 
9) L. Kassel, H. Abad, J. W. Gariand, P. M. Raccah, J. E. Potts, M. A. Haase and H. Cheng: Appl. Phys. Lett. 56 (1990) 42.

10) D. E. Aspnes: Optical properties of semiconductors (Handbook on Semiconductors II) ed. M. Balkanski (Amsterdam, North-Holland) Chap. 4A, p. 109.
11) W. C. Yeh, S. Chen, Y. S. Huang, C. H. Ho and K. K. Tiong: J. Phys.: Condens. Matter 12 (2000) 2183.

12) I. Vurgaftman, J. R. Meyer and L. R. Ram-Mohan: J. Appl. Phys. 89 (2001) 5815 . 